Article

\title{
Performance of Slow-Growing Male Muscovy Ducks Exposed to Different Dietary Levels of Quebracho Tannin
}

\author{
Annelisse Castillo ${ }^{1}{ }^{\oplus}$, Achille Schiavone ${ }^{1}\left(\mathbb{D}\right.$, Maria Grazia Cappai ${ }^{2}{ }^{\oplus}$, Joana Nery ${ }^{1, *}$, \\ Marta Gariglio ${ }^{1}$, Stefano Sartore ${ }^{1}$, Alessandro Franzoni ${ }^{1}$ and Margherita Marzoni ${ }^{3}$ \\ 1 Department of Veterinary Sciences, Università degli Studi di Torino, VIA Largo Paolo Braccini 2, \\ 10095 Grugliasco (TO), Italy; annelisse.castillogarrido@unito.it (A.C.); achille.schiavone@unito.it (A.S.); \\ marta.gariglio@unito.it (M.G.); stefano.sartore@unito.it (S.S.); alessandro.franzoni@unito.it (A.F.) \\ 2 Department of Veterinary Medicine, Università di Sassari, VIA Vienna 2, 07100 Sassari (SS), Italy; \\ mgcappai@uniss.it \\ 3 Department of Veterinary Sciences, Università di Pisa, VIA le delle Piagge 2, 56124 Pisa (PI), Italy; \\ margherita.marzoni@unipi.it \\ * Correspondence: joana.nery@unito.it; Tel.: +39-011-6709261
}

Received: 7 May 2020; Accepted: 2 June 2020; Published: 4 June 2020

Simple Summary: Different inclusion levels of Quebracho tannin (QT) in the diet of growing male Muscovy ducks of a slow-growing type were explored under free-range conditions. As a result of the dietary treatments tested in this trial, the growth performance or the total blood proteins were not affected. By contrast, dietary QT did not lead birds to produce less moist excreta, as observed in other bird species, as a desirable aspect for intensively raised poultry. A marked improvement in carcass yield was observed as a desirable economic trait in the extensive slow-type duck farming system.

\begin{abstract}
The study of the nutritional effects of tannins is complex due to the large chemical diversity; consequently, in poultry nutrition the biological responses may vary greatly. The aim of the present study was to evaluate the effect of different levels of dietary Quebracho tannins (QT) on growth and production performance in slow-growing type Muscovy ducks. For this purpose, a 42-d trial was carried out on 126 male ducks (42-d old at start), fed on three levels of dietary QT inclusion in the $\operatorname{diet}(0 \%$ as control diet, vs. $1.5 \%$ vs. $2.5 \%$ on an as fed basis). Birds were reared under free-range conditions. A linear increase in feed intake as a function of QT inclusion in the diet was observed $(p<0.05)$. No difference as to final body weight, overall average daily weight gain (ADG) and total feed conversion ratio (FCR) in relation to dietary treatments was observed. Carcass yields were positively improved in QT birds $(p<0.05)$. No adverse responses were recorded in total blood protein and liver weight. Dietary QT might be safely used up by to $2.5 \%$ in 42 - to 84 -d aged male Muscovy ducks.
\end{abstract}

Keywords: Muscovy duck; slow-growing; Quebracho tannin; growth performance; slaughter performance

\section{Introduction}

Tannins are plant secondary metabolites and represent the fourth most abundant group of secondary compounds of the plant kingdom, after cellulose, hemicellulose, and lignin [1]. Tannins are polyphenolic compounds, located mainly in vacuoles of the vegetal cell or in waxes, where they do not interfere with plant metabolism. They are found in many parts of the plant, such as fruits, leaves, bark, and wood [2], and in common foodstuffs, for example grapes, strawberries, blackberries, 
hazelnuts, cocoa, guarana. Feed such as sorghum grains, peas, fava beans, or legume trees like Acacia sp., Sesbania sp. also contain tannins [2,3], as well as acorns (Quercus spp.) and chestnuts (Castanea sativa), as spontaneous fruits of wood forest, chiefly for wild mammals and birds, but also domestic species [4-6]. Tannins exert various beneficial functions for the plant, depending on the tissue where they are found, such as regulating dormancy of seeds, contrasting pathogens acting as chemical barriers in roots and seeds, opposing predation of unripe fruits and leaves or preservation of the heartwood of conifers [2,3]. In some cases, tannins also attract insects towards flowers, thus helping in cross-pollination $[7,8]$.

Tannins belong to a heterogenous group of phenolic compounds, with different chemical structures but with a high affinity to bind and precipitate proteins. Tannins are mainly classified into three major groups: hydrolysable tannins, condensed tannins or proanthocyanidins, and phlorotannins. The first two groups are found in terrestrial plants, whilst phlorotannins are found in marine brown algae $[9,10]$. Hydrolysable tannins are susceptible to hydrolysis by acids, bases or esterases, being easily degraded and absorbed in the digestive tract of mammals and birds [11,12]. Condensed tannins are oligomeric or polymeric flavonoids with complex structures and high molecular weights. In contrast to hydrolysable tannins, only strong oxidative and acidic hydrolysis can depolymerize the condensed tannin structures that are also not susceptible to anaerobic enzyme degradation [13]. Among the condensed tannin sources tested in poultry diets, we can find grape seed extract [14-16], grape pomace [16,17], mimosa [18] and Quebracho [19-21].

The phenolic structure of tannins is responsible for the antioxidant activity, which finds application in different fields, such as food industry and animal feeding, medical and pharmaceutical sectors [22]. In view of such technological and biological properties, tannins are considered an attractive family of chemicals, due to their various application potentials in different fields. Nevertheless, the study of the nutritional effects of tannins is complicated by the large chemical diversity [23], and consequently the bird's responses may vary greatly. For instance, tannins were reported to act as anti-nutritional compounds in poultry diets, affecting productive performance due to a decrease in the feed intake and the digestibility of organic matter [24,25]. In contrast, it has been shown that depending on the bird's age, health and physiological status, beneficial effects might be obtained by combining a specific tannin group in the diet. In this regard, a positive influence on chicken growth performance [26], improved final body weight and feed efficiency in broilers [27], reduced cholesterol level in Leghorn hens eggs [28], potential anticoccidial agent in broilers [19] and anthelmintic activity in pheasants [20] were reported in the reference literature.

Between the period 1998 and 2018, the global duck meat production expanded significantly, from 2.62 to 4.46 million tons, respectively [29]. Asia is the main global producer, accounting for $83.0 \%$ of the total duck meat output, followed by Europe, with $11.7 \%$ in 2018 [29]. The rearing of ducks follows different production systems. In developed countries for example, they are mainly reared intensively, while in Asia extensive production is largely applied [30]. Within duck species, the Muscovy duck (Cairina moschata domestica, Linnaeus, 1758.) represents an important economic resource. Central and South America represent the origin of this species, but currently Muscovy duck production is located mainly in Europe, with France as the first producer, together with southeast Asia and Taiwan [31]. Muscovy ducks are omnivorous birds, and the natural diet is based on worms, insects, fish, amphibians, reptiles, plants, and fruits [32]. For this reason, tannins, being widely distributed in the plant kingdom, might reasonably be part of their natural diet. Quebracho tannin (Schinopsis sp.) as a biologically active compound has been documented in pheasants and chickens [20,21], but limited information is available about the effects in the diet of Muscovy ducks. Therefore, the aim of the present study was to evaluate the effect of two inclusion levels of purified Quebracho tannin (QT) in the diet of slow-growing male Muscovy ducks on growth and production performance. 


\section{Materials and Methods}

\subsection{Birds and Diets}

The experimental protocol (prot. no. 814715) was approved by the Bioethical Committee of the University of Turin (Italy). A feeding trial was carried out using one-hundred and twenty-six 42-d old males of Muscovy duck (Cairina moschata domestica, Linnaeus 1758), from a local slow-growing type, preserved since over 40 years at the Avian Conservation Center, which belongs to the Department of Veterinary Science of the University of Pisa (Italy). Birds were individually identified by a wing tag, weighted, and housed randomly in 18 roofed outdoor pens $(6 \times 3 \mathrm{~m})$ with sandy floor. Each pen housed seven animals.

Birds were allotted to the following experimental dietary groups: the control group (QT0) received the basal diet (Table 1); QT dietary groups received the same basal diet of QT0 with the inclusion of increasing QT levels at an amount of $1.5 \%$ and $2.5 \%$ on an as-fed basis, respectively. The QT is a commercial animal feed additive, extracted from the heartwood of Schinopsis sp., (MGM-S ${ }^{\circledR}$, Unitan SAICA, Buenos Aires, Argentina). This product is available as a fine powder with $58 \%$ tannins, $20 \%$ phlobaphenes, $14 \%$ non-tannic compounds and $8 \%$ water and a polymerization degree of $6-7$. Due to previous experiences in pheasants [20] and laying hens [21], the maximum safe level of QT to be used in diets for 42- to 84-d old Muscovy ducks is $2.5 \%$ on an as-fed basis, therefore, experimental groups were defined as group QT1.5 (2.6\% MGM-S powder on top, corresponding to $1.5 \%$ QT) and QT2.5 (4.3\% MGM-S powder on top, corresponding to 2.5\% QT). For each period, the basal diet met or exceeded NRC [33] duck's nutritional requirements and was formulated according to INRA [34] feed ingredient nutritional composition. Six replicates were assigned to each dietary treatment. Water and feed were supplied ad libitum. The chemical composition of the basal feed was analyzed in duplicate [35] and mean results are displayed in tables.

Table 1. Ingredients and chemical composition of basal diet.

\begin{tabular}{|c|c|c|}
\hline \multirow{2}{*}{ Ingredient (\%) } & \multicolumn{2}{|c|}{ Days } \\
\hline & $0-41$ & $42-84$ \\
\hline Corn meal & 64.10 & 67.30 \\
\hline Soybean meal & 16.00 & 10.00 \\
\hline Bran & 3.63 & 6.62 \\
\hline Corn gluten meal & 9.00 & 9.00 \\
\hline Soybean oil & 2.85 & 3.45 \\
\hline Dicalcium phosphate & 1.30 & 0.40 \\
\hline Calcium carbonate & 1.40 & 1.74 \\
\hline Sodium chloride & 0.25 & 0.25 \\
\hline Sodium bicarbonate & 0.20 & 0.20 \\
\hline DL-Met & 0.17 & 0.03 \\
\hline L-Lys & 0.39 & 0.30 \\
\hline Vitamin-mineral premix ${ }^{1}$ & 0.50 & 0.50 \\
\hline Choline cloride & 0.01 & 0.01 \\
\hline Optifos 250 bro $^{2}$ & 0.10 & 0.10 \\
\hline Avizyme $1500 x^{3}$ & 0.10 & 0.10 \\
\hline \multicolumn{3}{|l|}{ Chemical composition } \\
\hline Dry matter (DM), \% & 88.80 & 88.70 \\
\hline Crude protein, \% DM & 23.00 & 20.20 \\
\hline Ether extract, \% DM & 6.20 & 7.30 \\
\hline Neutral detergent fiber, \% DM & 12.90 & 12.70 \\
\hline Ash, \% DM & 7.80 & 6.50 \\
\hline Metabolizable energy, MJ/kg 4 & 12.53 & 12.77 \\
\hline
\end{tabular}

1 Supplied per kg of diet: Vit. A 62.5 IU; Vit. D3 17.5 IU; Vit. E $200 \mu g$; Vit. K $10 \mu g$; Biotin $1 \mu \mathrm{g}$; Thiamine $10 \mu \mathrm{g}$; Riboflavin $30 \mu$ g; Pantothenate $76.05 \mu \mathrm{g}$; Niacin $200 \mu$ g; Choline $3750 \mu \mathrm{g}$; Pyridoxine $20 \mu$ g; Folic acid $3.75 \mu \mathrm{g}$; Vit. B12

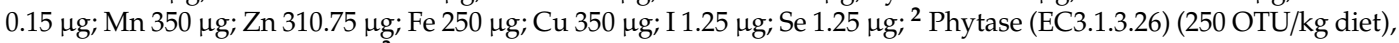
Huvepharma, Sofia, Bulgaria; ${ }^{3}$ Complex of Endo 1-4- $\beta$ - Xylanase (EC3.2.1.8) (256 U/kg), Subtilisin (EC3.4.21.62) (2560 U/kg diet) and $\alpha$-Amylase (EC3.2.1.1) (1472 U/kg diet), Danisco Animal Nutrition, Marlborough, Wiltshire, UK; ${ }^{4}$ Based on INRA [34] ingredient composition. 


\subsection{Growth Performance and Feed Conversion Efficiency}

Growth performance was evaluated biweekly during the age interval 42-84 d. Individual live body weight (LBW) and feed intake per pen were recorded to calculate the average daily weight gain (ADWG), the average daily feed intake (ADFI) and the feed conversion ratio (FCR).

The LBW in each group were fitted by using LAB Fit-Curve Fitting Software [36]. To trace the first part of the growth curve, individual LBW, previously recorded, of all birds at 0, 14 and 28-d age was considered. The same data were then used in each of the three growth curves (QT0, QT1.5 and QT2.5). The Gompertz function resulted the best fitting growth curve. The following equation describes the curve

$$
\mathrm{W}_{(\mathrm{t})}=\mathrm{W}_{¥} \mathrm{e}^{-\mathrm{e}^{-\mathrm{k}\left(\mathrm{t}-\mathrm{t}^{\circ}\right)}}
$$

where $W_{(t)}$ is the weight in grams of duck at time $t, W_{¥}$ is the asymptotic final LBW, $k$ is the constant expressing the rate of approach to $W_{¥}$, and $t^{\circ}$ is the age at the inflexion point where maximum rate of growth is achieved. The maximum rate of growth for each group was calculated by the differential equation of $1^{\circ}$ grade derived from Gompertz

$$
W^{\prime}(t)=k \cdot W_{¥} e^{-e^{-k \cdot\left(t-t^{\circ}\right)}-k \cdot\left(t-t^{\circ}\right)}
$$

The values of $W_{¥}, k$ and $t^{\circ}$ are the same described above for the growth curve, $W^{\prime}(t)$ is the daily weight increase in grams at time $t$ [37].

\subsection{Blood Sampling}

Blood samples from 24 birds ( $84 \mathrm{~d}$ old) from each dietary group (four birds/pen) were collected during slaughter procedures into $\mathrm{K}_{3}$ EDTA $2 \mathrm{~mL}$ vacuum tubes. Total blood protein (TBP) was determined according to Salamano et al. [38].

\subsection{Excreta Sampling and Dry Matter}

Excreta sample collection was carried out on 24 ducks (81 d old) per dietary treatment, following the procedure described by Gariglio et al. [39]. Briefly, the birds were kept and temporary housed (1 h) in cages (two birds in the same pen/cage). Excreta samples free from debris and foreign substances were collected from a tray placed under each cage. A total of 12 pooled samples per dietary treatment were collected at each sampling. The dry matter (DM) of excreta was determined according to AOAC [35].

\subsection{Slaughter Traits and Organs Evaluation}

Twelve birds ( $84 \mathrm{~d}$ old) per dietary treatment were randomly chosen and fasted for 12-h, to be finally weighed and slaughtered in a commercial abattoir. Birds were electrically stunned prior to bleeding. All bird carcasses were mechanically plucked after immersion in hot water. Evisceration was performed manually. Fresh absolute weight of liver and gizzard were determined, and relative weight expressed as percentage of live body weight (LBW). Head-neck and feet were removed and weighed separately; the respective relative weights were calculated as percentage of LBW. Hot weight of ready-to-cook carcass (RCC) was recorded and expressed as percentage of LBW. The small intestine and caeca were unfolded on a flat surface to obtain a fully extended viscera and allow linear length measurement; the respective lengths were expressed as $\mathrm{cm}$ per $100 \mathrm{~g}$ of LBW. Carcasses were stored in a cool chamber at $4 \pm 0.5{ }^{\circ} \mathrm{C}$ for $24 \mathrm{~h}$, then chilled RCC weights were determined and expressed as percentage of LBW. Finally, $m$. pectoralis and legs + thigs were separated from the RCC and weights were determined and expressed as percentage of LBW.

\subsection{Statistical Analysis}

The statistical analyses were performed using the SPSS software [40]. Shapiro-Wilk's test established normality or non-normality of distribution, and the assumption of equal variances was 
assessed by means of Levene's homogeneity of variance test. The experimental unit was the pen. The collected data were tested by means of one-way ANOVA, using polynomial contrasts to test the linear and quadratic responses to increases in the QT inclusion levels in the diet. For all tests, $p<0.05$ was considered statistically significant. Results were expressed as the mean and standard error of the means (SEM). A statistical trend was considered for $p<0.10$.

\section{Results}

\subsection{Growth Performance and Feed Conversion Ratio}

Throughout the experimental period, no mortality was experienced. Bird performance is summarized in Table 2. Overall LBW was not influenced by dietary treatments $(p>0.05)$, except for 70-d age, where a quadratic response was observed, and the minimum shown by QT1.5 group $(p<0.05)$.

Table 2. Growth performance of Muscovy ducks fed different levels of dietary Quebracho tannin (QT) from 42 to 84 -d age ( $n=6$ pens /treatment; 7 birds/pen).

\begin{tabular}{|c|c|c|c|c|c|c|c|}
\hline \multirow{2}{*}{ Items } & \multirow{2}{*}{ Age } & \multicolumn{3}{|c|}{$\operatorname{Diet}^{1}$} & \multirow{2}{*}{ SEM } & \multicolumn{2}{|c|}{$p$-Value } \\
\hline & & QT0 & QT1.5 & QT2.5 & & Linear & Quadratic \\
\hline \multirow{4}{*}{ LBW, g } & $42 \mathrm{~d}$ & 1699 & 1653 & 1676 & 14.6 & 0.541 & 0.259 \\
\hline & $56 \mathrm{~d}$ & 2703 & 2667 & 2635 & 16.4 & 0.094 & 0.949 \\
\hline & $70 \mathrm{~d}$ & 3408 & 3272 & 3330 & 20.6 & 0.126 & 0.023 \\
\hline & $84 \mathrm{~d}$ & 3787 & 3784 & 3789 & 19.1 & 0.956 & 0.913 \\
\hline \multirow{4}{*}{ ADWG, g/d } & $42-56 \mathrm{~d}$ & 71.8 & 72.4 & 68.5 & 0.77 & 0.084 & 0.147 \\
\hline & $56-70 \mathrm{~d}$ & 50.3 & 43.2 & 49.7 & 0.69 & 0.688 & 0.000 \\
\hline & $70-84 \mathrm{~d}$ & 27.0 & 36.5 & 32.8 & 0.75 & 0.001 & 0.000 \\
\hline & $42-84 \mathrm{~d}$ & 49.7 & 50.73 & 50.3 & 0.42 & 0.571 & 0.414 \\
\hline \multirow{4}{*}{ ADFI, g/d } & $42-56 \mathrm{~d}$ & 241 & 242 & 259 & 4.09 & 0.063 & 0.266 \\
\hline & $56-70 \mathrm{~d}$ & 232 & 227 & 269 & 7.44 & 0.010 & 0.036 \\
\hline & $70-84 \mathrm{~d}$ & 221 & 228 & 239 & 5.23 & 0.188 & 0.834 \\
\hline & $42-84 \mathrm{~d}$ & 231 & 232 & 256 & 4.75 & 0.014 & 0.116 \\
\hline \multirow{4}{*}{ FCR, g/g } & $42-56 \mathrm{~d}$ & 3.36 & 3.34 & 3.83 & 0.11 & 0.077 & 0.230 \\
\hline & $56-70 \mathrm{~d}$ & 4.61 & 5.28 & 5.46 & 0.20 & 0.090 & 0.536 \\
\hline & $70-84 \mathrm{~d}$ & 8.72 & 6.31 & 7.35 & 0.45 & 0.147 & 0.052 \\
\hline & $42-84 \mathrm{~d}$ & 4.68 & 4.58 & 5.11 & 0.11 & 0.114 & 0.172 \\
\hline
\end{tabular}

${ }^{1}$ QT0 = basal diet; QT1.5 and QT2.5 = the basal diet supplemented with QT at 1.5\% and 2.5\%, respectively. Note: SEM: standard error of the mean; LW: live body weight; ADWG: average daily weight gain; ADFI: average daily feed intake; FCR: feed conversion ratio.

Dietary QT negatively influenced ADWG during the middle part of the experimental period (56-70 d), showing a quadratic response $(p<0.01)$. The following period $(70-84 \mathrm{~d})$ instead, was positively influenced by QT, showing linear and quadratic responses with a minimum for QT0 group $(p<0.01)$.

The ADFI resulted directly proportional to the concentration of QT during the 56-70 period, showing a linear $(p<0.01)$ and a quadratic $(p<0.05)$ response. A linear increase $(p<0.05)$ was also observed when considering the whole experimental period (42-84 d). The FCR was not affected by dietary QT, considering both the single age periods, and the whole experimental period.

According to the calculated Gompertz parameters (Table 3), birds reached the inflection point when this trial began (42-d age). Curve parameters were close to each other (Table 3 ). 
Table 3. Estimated Gompertz growth parameters, inflection point, and goodness of fit in male Muscovy ducks fed different levels of dietary Quebracho tannin (QT).

\begin{tabular}{ccccccc}
\hline Diet $^{\mathbf{1}}$ & $\mathbf{W}_{\mathbf{¥}}(\mathbf{g})^{\mathbf{2}}$ & $\mathbf{K}^{\mathbf{3}}$ & $\mathbf{t}^{\circ} \mathbf{( d )} \mathbf{4}^{\mathbf{4}}$ & Maximum ADWG (g/d) & Weight at Inflection Point $\mathbf{( g )}$ & $\mathbf{R}^{\mathbf{2}}$ \\
\hline QT0 & 4470 & 0.0444 & 42 & 73 & 1677 & 0.98 \\
QT1.5 & 4425 & 0.0436 & 42 & 71 & 1662 & 0.99 \\
QT2.5 & 4475 & 0.0431 & 42 & 71 & 1657 & 0.98 \\
\hline
\end{tabular}

${ }^{1}$ QT0 = basal diet; QT1.5 and QT2.5 = the basal diet supplemented with QT at $1.5 \%$ and $2.5 \%$, respectively; ${ }^{2} \mathrm{~W}_{¥}$ $=$ asymptotic final LBW; ${ }^{3} \mathrm{k}=$ constant, expressing rate of approach to $\mathrm{W}_{\sharp} ;{ }^{4} \mathrm{t}^{\circ}=$ age at inflexion point; ADWG: average daily weight gain.

\subsection{Total Blood Proteins}

TBP in 84-d old birds are reported in Table 4. The dietary QT inclusion did not influence the level of TBP $(p>0.05)$. In birds, normal TBP concentrations ranged between $3.5-5.5 \mathrm{~g} / \mathrm{dl}$ [41]. Results in this study placed all birds within this range.

Table 4. Total blood protein (TBP) of Muscovy ducks fed different levels of dietary Quebracho tannin (QT) at 84-d age ( $n=6$ pen/treatment; 4 birds/pen).

\begin{tabular}{|c|c|c|c|c|c|c|c|}
\hline \multirow{2}{*}{ Items } & \multirow{2}{*}{ Age } & \multicolumn{3}{|c|}{$\operatorname{Diet}^{1}$} & \multirow{2}{*}{ SEM } & \multicolumn{2}{|c|}{$p$-Value } \\
\hline & & QT0 & QT1.5 & QT2.5 & & Linear & Quadratic \\
\hline ТВP \% & $84 \mathrm{~d}$ & 4.50 & 4.33 & 4.38 & 0.05 & 0.351 & 0.319 \\
\hline
\end{tabular}

${ }^{1} \mathrm{QT0}=$ basal diet; QT1.5 and QT2.5 = the basal diet supplemented with QT at 1.5\% and 2.5\%, respectively.

\subsection{Excreta Dry Matter Evaluation}

Excreta dry matter content in 81-d old birds is reported in Table 5. The dietary QT inclusion did not influence this parameter $(p>0.05)$.

Table 5. Excreta dry matter (DM) of Muscovy ducks fed different levels of dietary Quebracho tannin (QT) at 84-d age ( $n=6$ pen/treatment; 4 birds/pen).

\begin{tabular}{cccccccc}
\hline \multirow{2}{*}{ Items } & \multirow{2}{*}{ Age } & \multicolumn{3}{c}{ Diet $^{1}$} & \multirow{2}{*}{ SEM } & \multicolumn{2}{c}{$p$-Value } \\
\cline { 3 - 5 } \cline { 6 - 7 } & & QT0 & QT1.5 & QT2.5 & & Linear & Quadratic \\
\hline DM \% & $84 \mathrm{~d}$ & 45.3 & 47.4 & 49.0 & 1.89 & 0.448 & 0.949 \\
\hline
\end{tabular}

\subsection{Slaughter Traits}

Slaughter traits in 84-d old male Muscovy ducks are reported in Table 6. The dietary QT inclusion had no influence on the development of breast muscles and Legs + thigs $(p>0.05)$. Both RCC, hot and chilled, were positively influenced by dietary treatment, showing a linear $(p<0.05)$ and a quadratic response $(p<0.01)$. Dietary treatment affected linearly the Head-Neck mass, which decrease by the increase level of dietary QT $(p<0.05)$. All other slaughter traits were not affected by the dietary treatment $(p<0.05)$, except for the SIL, which evidenced a quadratic response, with the minimum value expressed by the lowest QT inclusion level $(p<0.01)$. 
Table 6. Slaughter traits of male Muscovy ducks fed different levels of Quebracho tannin (QT) from $42-\mathrm{d}$ to 84 -d age ( $n=6$ pens /treatment; 2 birds/pen).

\begin{tabular}{|c|c|c|c|c|c|c|c|}
\hline \multirow{2}{*}{ Items } & & \multicolumn{3}{|c|}{$\operatorname{Diet}^{1}$} & \multirow{2}{*}{ SEM } & \multicolumn{2}{|c|}{$p$-Value } \\
\hline & & QT0 & QT1.5 & QT2.5 & & Linear & Quadratic \\
\hline LBW & $\mathrm{g}$ & 3782 & 3793 & 3809 & 26.0 & 0.678 & 0.963 \\
\hline Breast & & 12.48 & 13.16 & 12.55 & 0.21 & 0.897 & 0.160 \\
\hline Legs + thighs & & 12.89 & 12.90 & 12.79 & 0.10 & 0.687 & 0.776 \\
\hline $\mathrm{RCC}_{\text {hot }}$ & & 59.6 & 62.6 & 61.0 & 0.33 & 0.019 & 0.000 \\
\hline $\mathrm{RCC}_{\text {chilled }}$ & & 58.5 & 61.5 & 60.1 & 0.32 & 0.014 & 0.000 \\
\hline Head-Neck & o of I RW & 10.3 & 10.1 & 9.5 & 0.16 & 0.035 & 0.531 \\
\hline Feet & $\%$ of LBW & 2.74 & 2.66 & 2.67 & 0.03 & 0.247 & 0.447 \\
\hline Liver & & 1.80 & 1.64 & 1.74 & 0.04 & 0.589 & 0.143 \\
\hline Heart & & 0.73 & 0.67 & 0.70 & 0.01 & 0.405 & 0.236 \\
\hline Gizzard & & 1.91 & 1.83 & 1.87 & 0.03 & 0.620 & 0.400 \\
\hline Intestine & & 4.87 & 4.71 & 4.76 & 0.13 & 0.740 & 0.691 \\
\hline SIL & $\mathrm{cm} / 100 \mathrm{~g}$ & 5.52 & 5.11 & 5.33 & 0.06 & 0.148 & 0.013 \\
\hline CL & LBW & 1.14 & 1.05 & 1.06 & 0.02 & 0.053 & 0.171 \\
\hline
\end{tabular}

${ }^{1}$ QT0 = basal diet; QT1.5 and QT2.5 = the basal diet supplemented with QT at $1.5 \%$ and $2.5 \%$, respectively. Note: SEM: standard error of the mean; LBW: live body weight; RCC: ready to cook carcass; SIL: small intestine length; CL: caeca length.

\section{Discussion}

The increasing interest in the use of some plant-derived molecules with biological properties offers the chance to exploit several beneficial effects due to their numerous properties [9,42]. Against this background, more information seems to be needed about their safe use in different species, to achieve the desired effects. From this perspective, and due to previous experiences in pheasant [20] and pullets [21], the maximum safe QT level to be used in diets for 42 to 84-d old Muscovy ducks was set to $2.5 \%$, on an as-fed basis. At this QT amount, no adverse effects were observed in birds of this trial, as supported by the TBP levels and liver weight. On the contrary, in a previous study involving slow-growing pullets [21], adverse effects were observed in 35-d old birds fed 3\% dietary tannins supplementation, resulting in $2 \%$ of QT as the safe inclusion level. It is widely known that tannins bind and precipitate proteins, which compromises absorption at intestinal level. This situation might lead to protein deficiency in the bloodstream [9]. Ducks in this study fed on QT diets did not exhibit a decrease in TBP circulating value. Additionally, our results agree with those obtained by Gariglio et al. [43] in 50-d old female Muscovy ducks.

As to liver, weight variations can be useful signs of the presence of hepatic injuries [44]. In this trial, liver weights in QT birds were similar across birds from all experimental groups, at each QT inclusion rate tested. Furthermore, at the end of the experimental period, birds of QT1.5 and QT2.5 groups reached a similar LBW at slaughter. As demonstrated also by the growth curve trends, which were almost identical, it can be stated that the tested amounts of QT included in the diet did not negatively impact the production performance of slow-growing male Muscovy ducks. In agreement with other authors, who reported duck's growth curves using Gompertz model [45,46], we also found this model as the best fitting in our trials $\left(R^{2}=0.98-0.99\right)$.

According to the QT inclusion levels tested, a different reaction in birds was noted regarding feed intake. Birds of the QT1.5 group needed a longer time to get used to the change in diet, as demonstrated during the first days after dietary QT inclusion with an evident decrease in the ADWG and ADFI. Birds of the QT2.5 group, instead, seemed to cope with the QT by increasing the ADFI, which was higher during the whole experimental period. Such differences in response to different levels of dietary QT inclusion might be explained by the natural feeding behavior of the Muscovy duck, for which a large part of foraging activity takes place in fresh water [32]. In captivity, when ducks find a hard, unusual or an unknown tasty food, they use to soak it into water in order to render it "eatable". It could be argued that such behavior of soaking feeds was performed with the purpose of expressing the innate feeding 
behavior. With the increasing QT, the feed taste becomes reasonably astringent and birds tended to put the feed into the water with a consistent higher consumption. In QT1.5 group, instead, astringency perception presumably following a lower QT inclusion amount, might have not led birds to adopt coping solutions, for which a lower feed intake was also observed. Elkin et al. [47] reported no growth depression in ducks fed sorghum rich in tannins. Same authors also suggested that the innate feeding behavior of ducks could be a key factor in the interpretation of such a response.

In this trial, the FCR calculated throughout the experimental period and the final LBW were not affected by dietary QT at both tested levels. However, in QT1.5 birds, an initial retarded growth occurred, with a successive compensatory growth, evident especially during the last 15 days (70-84). This compensatory growth trend was also reported by other authors in broilers reared under feeding restriction, followed by ad libitum feed provision [48]. A decrease in growth rate, lower LBW, lower ADFI and higher FCR were reported in male Muscovy ducks fed low- or high-tannin-sorghum diets [49]. These authors also reported no differences in these parameters in Muscovy males fed on diets 50/50 high/low tannin-sorghum enriched with L-Methionine, attributing the reduction in the negative effect of tannin on protein availability to this amino acid.

Unexpectedly, the use of dietary QT did not lead birds to excrete dryer droppings as observed in other bird species, namely chickens [21] and pheasants [20], but rather the opposite effect could be observed. This result might be attributed to the high daily water intake in ducks, which did not allow the QT to have a "drying" effect. As some authors reported, $800 \mathrm{ml} / \mathrm{d}$ was the water intake in 14 to 42-d old Pekin ducks [50], while in 20-week old White Leghorn hens the intake was 228 ml/d [51], thus, threefold lower than the intake in ducks.

The dietary QT did not negatively affect carcass traits in 84-d old male ducks, rather, it led to improved yields in both the hot and chilled RCC of QT birds, which was heavier than QT0. Higher RCC yields were also reported in male Muscovy ducks of the same strain [52], fed on diets with 50/50 high/low tannin-sorghum [48]. In slow-growing chickens, instead, the RCC yield pointed out no differences [21], as well as in broiler chickens [26] and in female pheasants [20].

Wild birds feeding on diets rich in tannins developed larger intestines and caeca, and heavier gizzards [53], however, this could be also induced by a different physical form of natural feeding sources, rich also in more fibrous nutrient content [54]. In this trial, small intestine length was influenced by the diet. Nevertheless, this influence resulted in the opposite to what we would have expected, with QT1.5 birds having a shorter small intestine. In contrast, no effect was observed in growing female pheasants after a 60-d QT diet [20], and longer small intestine length after a 4-week trial in adult grey partridges was observed by Liukkonen-Anttila et al. [53]. Further research is needed to clarify this result.

\section{Conclusions}

In view of the parameters tested in this feeding trial, no adverse effects on health nor on the production performance of birds could be observed. The increasing amount of QT in the diet was found to be linked to the higher intake of feed by the ducks of the different dietary groups, likely due to the capability of dietary quebracho tannins to stimulate the innate feeding behavior. Excreta quality was not influenced by the amount of tannins in the diet, probably due to a conserved water consumption via soaked feed. Finally, the slaughtering performance was satisfactory and improved by QT supplementation, thus the inclusion up to $2.5 \%$ of Quebracho tannins in the diet can be safely used in slow-growing type male Muscovy ducks.

Author Contributions: Conceptualization, A.C., A.S. and M.M.; methodology, A.C., A.S. and M.M.; software analysis, A.C., M.G. and J.N.; validation, A.C., M.G., M.G.C., M.M. and S.S.; formal analysis, A.C., A.S., M.G. and M.M.; investigation A.C., A.F., A.S., J.N. and M.M.; data curation, A.C., A.F., M.M. and S.S.; writing-original draft preparation, A.C., A.S. and M.G.C.; writing-review and editing, A.C., A.S., J.N., M.G.C. and M.M.; supervision, A.S.; funding acquisition, J.N. and M.M. All authors have read and agreed to the published version of the manuscript. 
Funding: Appreciation is expressed to UNITAN S.A.I.C.A. for funding this research project and providing the commercial product: MGM-S. This study was supported by Ministero dell'Istruzione, dell'Universitaà e della Ricerca (MIUR) under the programme "Dipartimenti di Eccellenza ex L.232/2016" to the Department of Veterinary Science, University of Turin (Italy).

Acknowledgments: We thank D. Tedeschi (Laboratorio Tedeschi, Veterinary Clinical Analysis) for performing plasma analysis.

Conflicts of Interest: The authors declare no conflict of interest.

\section{References}

1. Chowdhury, S.P.; Khanna, S.; Verma, S.C.; Tripathi, A.K. Molecular diversity of tannic acid degrading bacteria isolated from tannery soil. J. Appl. Microbiol. 2004, 97, 1210-1219. [CrossRef] [PubMed]

2. Haslam, E.; Cai, Y. Plant polyphenols (vegetable tannins) - Gallic acid metabolism. Nat. Prod. Rep. 1994, 11, 41-66. [CrossRef] [PubMed]

3. Butler, L.G. Effects of condensed tannin on animal nutrition. In Chemistry and Significance of Condensed Tannins; Hemingway, R.W., Karchesy, J.J., Branham, S.J., Eds.; Springer: Boston, MA, USA, 1989; pp. 391-402.

4. Cappai, M.G.; Wolf, P.; Pinna, W.; Kamphues, J. Pigs use endogenous proline to cope with acorn (Quercus pubescens Willd.) combined diet high in hydrolysable tannins. Livest. Sci. 2013, 155, 316-322. [CrossRef]

5. Cappai, M.G.; Wolf, P.; Rust, P.; Pinna, W.; Kamphues, J. Raw hulled shredded acorns from Downy Oak (Quercus pubescens) in the diet of pigs: Effects on digestibility and faeces characteristics. J. Anim. Physiol. Anim. Nutr. 2013, 97, 1-5. [CrossRef] [PubMed]

6. Cappai, M.G.; Wolf, P.; Pinna, W.; Kamphues, J. The bilateral parotidomegaly (hypertrophy) induced by acorn consumption in pigs is dependent on individual's age but not on intake duration. Livest. Sci. 2014, 167, 263-268. [CrossRef]

7. Bovy, A.; Schijlen, E.; Hall, R.D. Metabolic engineering of flavonoids in tomato (Solanum lycopersicum): The potential for metabolomics. Metabolomics 2007, 3, 399-412. [CrossRef]

8. Winkel-Shirley, B. Biosynthesis of flavonoids and effects of stress. Curr. Opin. Plant. Biol. 2002, 5, $218-223$. [CrossRef]

9. Huang, Q.Q.; Liu, X.L.; Zhao, G.Q.; Hu, T.M.; Wang, Y.X. Potential and challenges of tannins as an alternative to in-feed antibiotics for farm animal production. Anim. Nutr. 2018, 4, 137-150. [CrossRef]

10. Yang, C.B.; Chowdhury, M.A.K.; Hou, Y.Q.; Gong, J. Phytogenic compounds as alternatives to in-feed antibiotics: Potentials and challenges in application. Pathogens 2015, 4, 137-156. [CrossRef]

11. Dollahite, J.W.; Pigeon, R.F.; Camp, B.J. The toxicity of gallic acid, pyrogallol, tannic acid, and Quercus havardi in the rabbit. Am. J. Vet. Res. 1962, 23, 1264-1267.

12. Mcleod, M.N. Plant tannins-their role in forage quality. Nutr. Abstr. Rev. 1974, 44, 803-812.

13. McSweeney, C.S.; Palmer, B.; McNeill, D.M.; Krause, D.O. Microbial interactions with tannins: Nutritional consequences for ruminants. Anim. Feed Sci. Technol. 2001, 91, 83-93. [CrossRef]

14. Ebrahimzadeh, S.K.; Navidshad, B.; Farhoomand, P.; Aghjehgheshlagh, F.M. Effects of exogenous tannase enzyme on growth performance, antioxidant status, immune response, gut morphology and intestinal microflora of chicks fed grape pomace. S. Afr. J. Anim. Sci. 2018, 48, 2-18. [CrossRef]

15. Farahat, M.H.; Abdallah, F.M.; Ali, H.A.; Hernandez-Santana, A. Effect of dietary supplementation of grape seed extract on the growth performance, lipid profile, antioxidant status and immune response of broiler chickens. Animal 2017, 11, 771-777. [CrossRef]

16. Viveros, A.; Chamorro, S.; Pizarro, M.; Arija, I.; Centeno, C.; Brenes, A. Effects of dietary polyphenol-rich grape products on intestinal microflora and gut morphology in broiler chicks. Poult. Sci. 2011, 90, 566-578. [CrossRef]

17. Chamorro, S.; Viveros, A.; Rebole, A.; Rica, B.D.; Arija, I.; Brenes, A. Influence of dietary enzyme addition on polyphenol utilization and meat lipid oxidation of chicks fed grape pomace. Food Res. Int. 2015, 73, 197-203. [CrossRef]

18. Iji, P.A.; Khumalo, K.; Slippers, S.; Gous, R.M. Intestinal function and body growth of broiler chickens on maize-based diets supplemented with mimosa tannins and a microbial enzyme. J. Sci. Food. Agric. 2004, 84, 1451-1458. [CrossRef] 
19. Cejas, E.; Pinto, S.; Prosdocimo, F.; Batalle, M.; Barrios, H.; Tellez, G.; De Franceschi, M. Evaluation of Quebracho Red Wood (Schinopsis lorentzii) Polyphenolic Vegetable Extract for the Reduction of Coccidiosis in Broiler Chicks. Int. J. Poult. Sci. 2011, 10, 344-349. [CrossRef]

20. Marzoni, M.; Castillo, A.; Romboli, I. Dietary inclusion of Quebracho (Schinopsis lorentzii) tannins on productive performances of growing pheasant females. Ital. J. Anim. Sci. 2005, 4, 507-509. [CrossRef]

21. Marzoni, M.; Castillo, A.; Franzoni, A.; Nery, J.; Fortina, R.; Romboli, I.; Schiavone, A. Effects of Dietary Quebracho Tannin on Performance traits and parasite load in an Italian Slow-growing Chicken (White Livorno breed). Animals 2020, 10, 684. [CrossRef]

22. Park, M.; Cho, H.; Jung, H.; Lee, H.; Hwang, K.T. Antioxidant and anti-inflammatory activities of tannin fraction of the extract from black raspberry seeds compared to grape seeds. J. Food Biochem. 2014, 38, 259-270. [CrossRef]

23. Mueller-Harvey, I. Unravelling the conundrum of tannins in animal nutrition and health. J. Sci. Food Agric. 2006, 86, 2010-2037. [CrossRef]

24. Longstaff, M.A.; McNab, J.M. The effect of concentration of tannin-rich bean hulls (Vicia faba L.) on activities of lipase (EC 3.1.1.3) and $\alpha$-amylase (EC 3.2.1.1) in digesta and pancreas and on the digestion of lipid and starch by young chicks. Br. J. Nutr. 2007, 66, 139. [CrossRef] [PubMed]

25. Vilarino, M.; Metayer, J.P.; Crepon, K.; Duc, G. Effects of varying vicine, convicine and tannin contents of faba bean seeds (Vicia faba L.) on nutritional values for broiler chicken. Anim. Feed Sci. Technol. 2009, 150, 114-121. [CrossRef]

26. Schiavone, A.; Guo, K.; Tassone, S.; Gasco, L.; Hernandez, E.; Denti, R.; Zoccarato, I. Effects of a natural extract of chestnut wood on digestibility, performance traits, and nitrogen balance of broiler chicks. Poult. Sci. 2008, 87, 521-527. [CrossRef] [PubMed]

27. Starcevic, K.; Krstulovic, L.; Brozic, D.; Mauric, M.; Stojevic, Z.; Mikulec, Z.; Bajic, M.; Masek, T. Production performance, meat composition and oxidative susceptibility in broiler chicken fed with different phenolic compounds. J. Sci. Food Agric. 2015, 95, 1172-1178. [CrossRef]

28. Minieri, S.; Buccioni, A.; Serra, A.; Galigani, I.; Pezzati, A.; Rapaccini, S.; Antongiovanni, M. Nutritional characteristics and quality of eggs from laying hens fed on a diet supplemented with chestnut tannin extract (Castanea sativa Miller). Br. Poult. Sci. 2016, 57, 824-832. [CrossRef]

29. FAO. Livestock primary. Available online: http://www.fao.org/faostat/en/\#data/QL (accessed on 15 May 2020).

30. Baeza, E. Quality and processing of duck meat products. In Proceedings of the 6th World Waterfowl Conference, Taipei, Taiwan, 22-25 October 2017; Available online: https://prodinra.inra.fr/record/410851 (accessed on 15 May 2020).

31. Swayne, D.E. Avian Influenza; Blackwell Publishing Ltd.: Oxford, UK, 2008; p. 61.

32. Rodenburg, T.B.; Bracke, M.B.M.; Berk, J.; Cooper, J.; Faure, J.M.; Guemene, D.; Guy, G.; Harlander, A.; Jones, T.; Knierim, U.; et al. Welfare of ducks in European duck husbandry systems. Worlds Poult. Sci. J. 2005, 61, 633-646. [CrossRef]

33. Council, N.R. Nutrient Requirements of Poultry, 9th Revised ed.; The National Academies Press: Washintong, DC, USA, 1994.

34. Sauvant, D.; Perez, J.M.; Tran, G. Tables INRA-AFZ de Composition et de Valeur Nutritive des Matières Premières Destinées Aux Animaux d'élevage: Porcs, Volailles, Bovins, Ovins, Caprins, Lapins, Chevaux, Poissons. 2ème Edition Revue et Corrigée; QUAE: Paris, France, 2004; p. 301.

35. A.O.A.C. Official Methods of Analysis, 17th ed.; Association of Official Analytical Chemist: Arlington, VA, USA, 2000.

36. Silva, W.P.; Silva, C.M.D.P.S. LAB Fit Curve Fitting Software-Nonlinear regression and treatment of data program, 7.2.50; 1999-2016. Available online: http://labfit.net/ (accessed on 15 May 2020).

37. Soft Warehouse, Derive for Windows-A Mathematical Assistant, 4.11; Soft Warehouse, Inc.: Chigago, IL, USA, 1988-1996.

38. Salamano, G.; Mellia, E.; Tarantola, M.; Gennero, M.S.; Doglione, L.; Schiavone, A. Acute phase proteins and heterophil-lymphocyte ratio in laying hens in different housing systems. Vet. Rec. 2010, 167, 749-751. [CrossRef]

39. Gariglio, M.; Dabbou, S.; Biasato, I.; Capucchio, M.T.; Colombino, E.; Hernandez, F.; Madrid Sanchez, J.; Martinez, S.; Gai, F.; Caimi, C.; et al. Nutritional effects of the dietary inclusion of partially defatted Hermetia illucens larva meal in Muscovy duck. J. Anim. Sci. Biotechnol. 2019, 10, 37. [CrossRef] 
40. IBM Corp. IBM SPSS Statistics for Windows, Version 25.0; IBM Corp.: Armonk, NY, USA, 2017.

41. Scanes, C.G. Protein metabolism. In Sturkie's Avian Physiology; Scanes, C., Ed.; Academic Press, Elsevier Inc.: Waltham, MA, USA, 2015; pp. 455-468.

42. Marzoni, M.; Chiarini, R.; Castillo, A.; Romboli, I.; De Marco, M.; Schiavone, A. Effects of dietary natural antioxidant supplementation on broiler chicken and Muscovy duck meat quality. Anim. Sci. Pap. Rep. 2014, 32, 359-368.

43. Gariglio, M.; Dabbou, S.; Crispo, M.; Biasato, I.; Gai, F.; Gasco, L.; Piacente, F.; Odetti, P.; Bergagna, S.; Placha, I.; et al. Effects of the Dietary Inclusion of Partially Defatted Black Soldier Fly (Hermetia illucens) Meal on the Blood Chemistry and Tissue (Spleen, Liver, Thymus, and Bursa of Fabricius) Histology of Muscovy Ducks (Cairina moschata domestica). Animals 2019, 9, 307. [CrossRef]

44. Cattley, R.C.; Cullen, J.M. Liver and Gall Bladder. In Haschek and Rousseaux's Handbook of Toxicologic Pathology, 3rd ed.; Academic Press: Salt Lake City, UT, USA, 2013; Volumes 1-3, pp. 1509-1566. [CrossRef]

45. Baeza, E.; De Carville, H.; Salichon, M.R.; Marche, G.; Leclercq, B. Effects of selection, over three and four generations, on meat yield and fatness in Muscovy ducks. Br. Poult. Sci. 1997, 38, 359-365. [CrossRef]

46. Arai, K.; Sato, T.; Goto, N. Growth curves of Spotbill ducks, Anas poecilorhyncha zonorhyncha Swinhoe, in captivity. Jpn. Poult. Sci. 1983, 20,337. [CrossRef]

47. Elkin, R.G.; Rogler, J.C.; Sullivan, T.W. Comparative effects of dietary tannins in ducks, chicks, and rats. Poult. Sci. 1990, 69, 1685-1693. [CrossRef]

48. Rahimi, S.; Seidavi, A.; Sahraei, M.; Blanco, F.P.; Schiavone, A.; Marin, A.L.M. Effects of feed restriction and diet nutrient density during re-alimentation on growth performance, carcass traits, organ weight, blood parameters and the immune response of broilers. Ital. J. Anim. Sci. 2015, 14, 583-590. [CrossRef]

49. Bagliacca, M.; Paci, G.; Marzoni, M.; Biagi, G.; Avanzi, C.F. Effect of sorghum in diets for ducks. Arch. Tierzucht 1997, 40, 277-286.

50. Veltmann, J.R.; Sharlin, J.S. Influence of water deprivation on water consumption, growth, and carcass characteristics of ducks. Poult. Sci. 1981, 60, 637-642. [CrossRef]

51. Parker, D.B.; Brown, M.S. Water consumption for livestock and poultry production. In Encyclopedia of Water Science; Howell, B.A.S.A.T.A., Ed.; Marcel Dekker Inc.: New York, NY, USA, 2003; pp. 588-591.

52. Schiavone, A.; Romboli, I.; Chiarini, R.; Marzoni, M. Influence of dietary lipid source and strain on fatty acid composition of Muscovy duck meat. J. Anim. Physiol. Anim. Nutr. 2004, 88, 88-93. [CrossRef] [PubMed]

53. Liukkonen-Anttila, T.; Kentala, A.; Hissa, R. Tannins-A dietary problem for hand-reared grey partridge Perdix perdix after release? Comp. Biochem. Physiol. C-Toxicol. Pharmacol. 2001, 130, 237-248. [CrossRef]

54. Cappai, M.G.; Arru, G.; Manconi, M.; Muzzeddu, M.; Pinna, W. Morphometric traits of gizzard in relation to feeding habits of wild Sardinian partridges (Alectoris barbara barbara, Bonnaterre, 1790) with particular regard to clast selection. J. Anim. Physiol. Anim. Nutr. 2017, 101, 421-426. [CrossRef] [PubMed] 